ИЗВЕСТИЯ АКАДЕМИИ НАУК ЭСТОНСКОИ ССР. ФИЗИКА МАТЕМАТИКА

PROCEEDINGS OF THE ACADEMY OF SCIENCES OF THE ESTONIAN SSR, PHYSICS * MATHEMATICS

$1987,36,2$

удК $535.37 ; 548.736$

Светлана ЗАЗУБОВИЧ, В. НАГИРНЫИ, Т. СООВНК

\title{
КИНЕТИКА ЗАТУХАНИЯ И ПОЛЯРИЗАЦИЯ ТРИПЛЕТНОЙ ЛЮМИНЕСЦЕНЦИИ ЦЕНТРОВ ДВУХВАЛЕНТНОГО ОЛОВА В ЩгК
}

\author{
(Представил Ч. Лущцк)
}

\section{1. Введение}

Основными центрами люминесценции в щелочногалоидных кристаллах (ЩГК), активированных оловом, являются ионы $\mathrm{Sn}^{2+}$, ассоциированные с катионной вакансией $v_{c}^{-}$(анизотропные $\mathrm{Sn}^{2+} v_{c}^{-}$-центры). Путем диссоциации этих диполей в небольшом количестве удается создать изотропные $\mathrm{Sn}^{2+}$-центры [ $\left.{ }^{1}\right]$.

На энергетической поверхности адиабатического потенциала (ЭПАП) как синглетного, так и триплетного возбужденных состояний ртутеподобного центра в ЩГК в общем случае могут сосуществовать три тетрагональных $(T)$ и четыре тригональных $(X)$ ян-теллеровских минимума. Излучение центров двухвалентного олова обусловлено преимущественно * электронными переходами из тетрагональных $(X, Y, Z)$ минимумов ЭПАП [1-4]. У изотропных $\mathrm{Sn}^{2+}$-центров ян-теллеровские $X$-, $Y$ - и $Z$-минимумы энергетически эквивалентны. У $\mathrm{Sn}^{2+v_{c}^{-}-ц е н т р о в ~} Z$-минимумы как синглетного, так и триплетного возбужденных состояний лежат ниже, чем $X, Y\left[{ }^{6}\right]$ (см. рис. $\left.1, \sigma\right)$, из-за $v_{c}^{-}$около $\mathrm{Sn}^{2+}(z-$ ось сим-

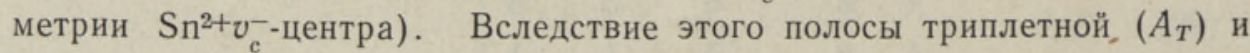
синглетной $\left(C_{T}\right)$ люминесценций $\mathrm{Sn}^{2+} v_{c}^{-}$-центров расщеплены на коротковолновую $A_{2}\left(C_{2}\right)$ (переходы из $X-, Y$-минимумов) и длинноволновую $A_{1}\left(C_{1}\right)$ (переходы из $Z$-минимумов) $\left[{ }^{2,3}\right]$.

Спин-орбитальное взаимодействие приводит к расщеплению каждого ян-теллеровского минимума триплетного состояния на излучательный дублет и расположенный под ним метастабильный синглет. В излучательных $\left(X_{y, z}, Y_{x, z}, Z_{x, y}\right)$ минимумах направление спиновой функции перпендикулярно, а в метастабильных $\left(X_{x}, Y_{y}, Z_{z}\right)$ - параллельно оси янтеллеровского искажения. Такая общая структура возбужденных состояний ртутеподобных центров, теоретически изученная в $\left[{ }^{7,8}\right]$, подтверждена многочисленными экспериментами (см., напр., обзоры $\left.\left[{ }^{9-11}\right]\right)$.

Однако при низких температурах в температурных зависимостях степени поляризации $A_{T}$-излучения $\mathrm{Sn}^{2+}$ и $\mathrm{Sn}^{2+} v_{c}^{-}$-центров нами $\left[{ }^{9}\right]$ и позднее в $\left[{ }^{12}\right]$ наблюдался ряд интересных особенностей, для объяснения которых потребовалось исследование более тонкой структуры ЭПАП

* Излучение $\mathrm{KCl}-\mathrm{Sn}$ с $\left|P_{\alpha=45^{\circ}}\right| \sim 2-3 \%$, перекрывающееся $A_{T}$-излучением, наблюдалось в $\left.{ }^{5}\right]$ и интерпретировано как $A_{X}$-излучение. Однако на основании анализа результатов [5] и собственных исследований этого излучения мы не можем с уверенностью заключить, что оно принадлежит основным $\mathrm{Sn}^{2+} v_{c}-$-центрам. 

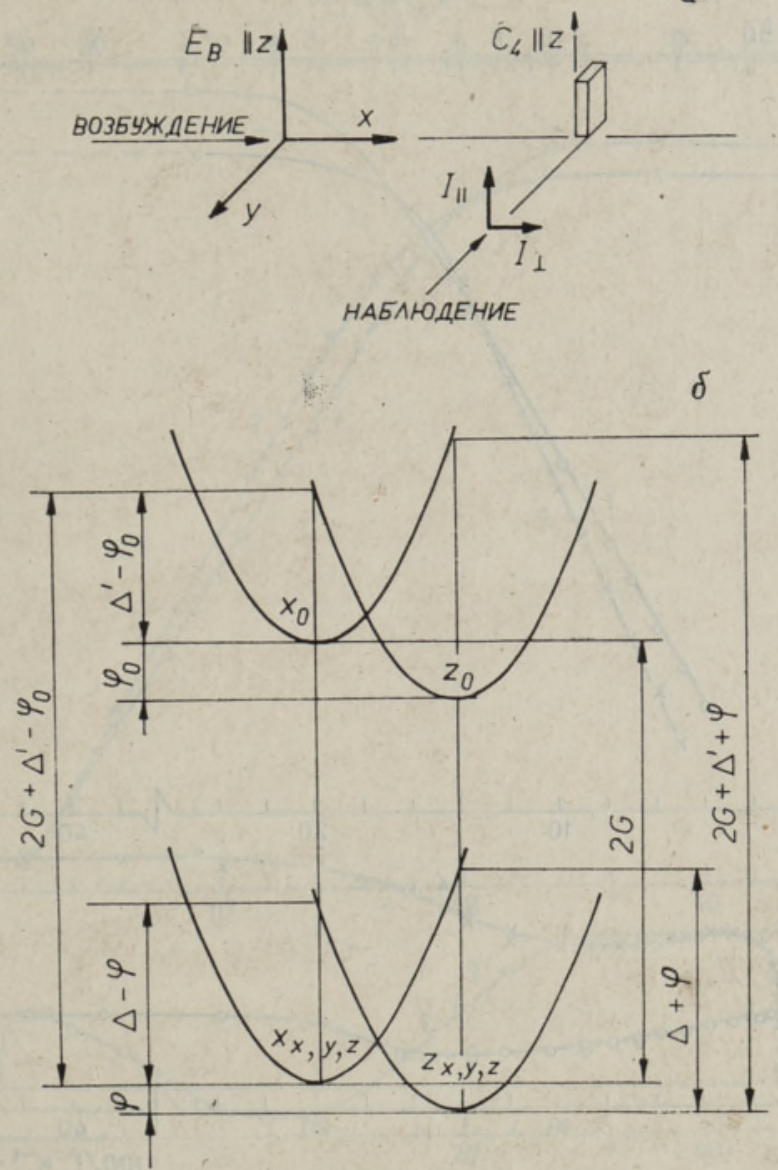

Рис. 1. Условия возбуждения и наблюдения при исследовании поляризации излучения (a) и взаимное расположение $X(Y)$ - и $Z$-минимумов синглетного и триплетного состояний $\mathrm{Sn}^{2+} v_{e}^{-}$-центров (б).

триплетного возбужденного состояния $\mathrm{Sn}^{2+} v_{c}^{-}$-центров в ЩГК и происходящих в нем процессов.

С этой целью в настоящей работе нами детально исследованы кинетические и поляризационные характеристики $A_{T}$-излучения изотропных $\mathrm{Sn}^{2+}$-центров и отдельных $\left(A_{1}\right.$ и $\left.A_{2}\right)$ компонентов $A_{T}$-излучения анизотропных $\mathrm{Sn}^{2+v_{c}^{-}-ц е н т р о в ~ в ~ и н т е р в а л е ~ т е м п е р а т у р ~ о т ~} 1,7$ до $40 \mathrm{~K}$.

\section{2. Кинетика затухания $A_{T}$-излучения}

В затухании $A_{T}$-излучения $\mathrm{Sn}^{2+}$ - и $\mathrm{Sn}^{2+v_{c}^{-}}$-центров во всех ЩГК быстрый компонент, обусловленный переходами из излучательных минимумов до установления термического равновесия с метастабильным, отсутствует (см. также $[4,9,13-15])$ даже при предельно низких температуpax $(0,5 \mathrm{~K})$. Это свидетельствует о том, что излучательные минимумы этих центров первоначально не заселяются, а уже на начальных стадиях релаксации в излучательные минимумы происходят безызлучательные переходы в метастабильное состояние. Излучательные минимумы $\mathrm{Sn}^{2+} v_{c}^{-}$и $\mathrm{Sn}^{2+}$-центров заселяются только в результате переходов из 


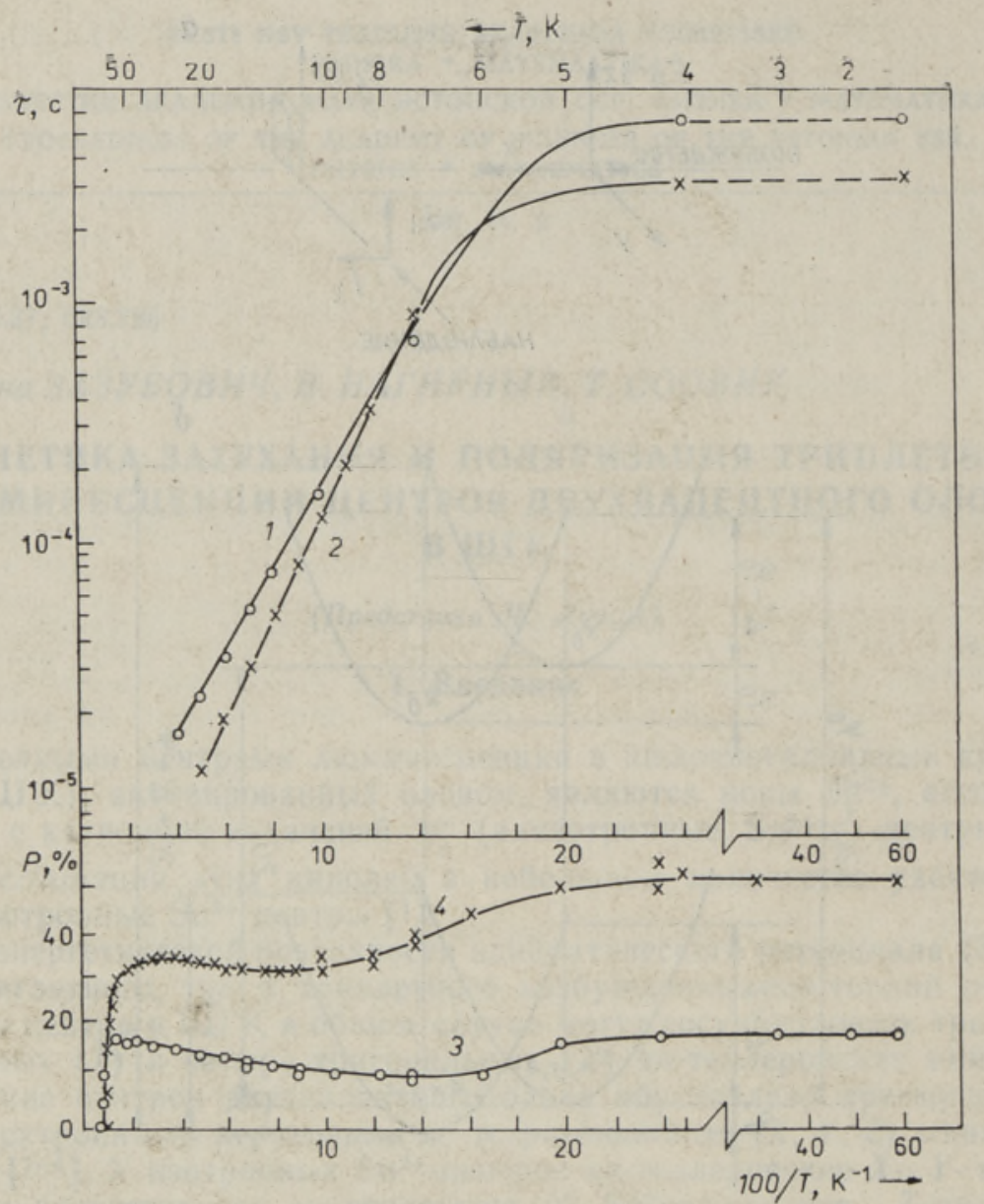

Рис. 2. Зависимости времен затухания $(1,2)$ и степеней поляризации $(3,4) A_{T}$-излучения $\mathrm{Sn}^{2+}$-центров в кристаллах $\mathrm{KCl}-\mathrm{Sn}(1,3)$ и $\mathrm{KBr}-\mathrm{Sn}(2,4)$ от температуры. $h v_{\mathrm{B}}=3,4$ э $\mathrm{B}$.

метастабильных минимумов. Температурные зависимости времени затухания $\tau$ равновесного компонента качественно аналогичны у всех исследованных $\mathrm{Sn}^{2+}$ - и $\mathrm{Sn}^{2+} v_{c}^{-}$-центров (рис. $\left.2-5\right)$.

Детальное исследование характеристик изотропных $\mathrm{Sn}^{2+}$-центров

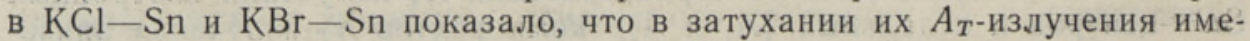
ется только один медленный компонент **. Длительность его $\tau$ экспоненциально уменьшается с ростом температуры в области $7-30 \mathrm{~K}$ (рис. 2). При $T<4,2 \mathrm{~K} \tau$ постоянно и составляет $\tau_{1}=5,5$ мс для $\mathrm{KCl}-\mathrm{Sn}$ и 3 мс для $\mathrm{KBr}-\mathrm{Sn}$. При этих температурах излучение обусловлено радиационным распадом метастабильных минимумов с вероятностью $\gamma_{1}=\tau_{1}^{-1}$. При $T>40 \mathrm{~K} \tau$ также не зависит от температуры и принимает значение $\tau_{2}=4-5$ мкс, которое определяется как вероятностью радиационного распада излучательных минимумов $\left(\gamma_{2}\right)$, так и вероятностью безызлучательных переходов из этих минимумов $\left(p_{0}\right)$.

У а н и зотропных $\mathrm{Sn}^{2+} v_{c}^{-}$-центров во всех исследованных кристаллах как времена затухания, так и их температурные зависимости различаются для $A_{1}$ - и $A_{2}$-полос излучения (рис. $\left.3-5\right)$. При этом для каждой

** Второй медленный компонент в затухании излучения $\mathrm{Sn}^{2+}$-центров в $\mathrm{KBr}-\mathrm{Sn}$. получен в $\left[{ }^{9}\right]$ из-за некорректного учета фона. 


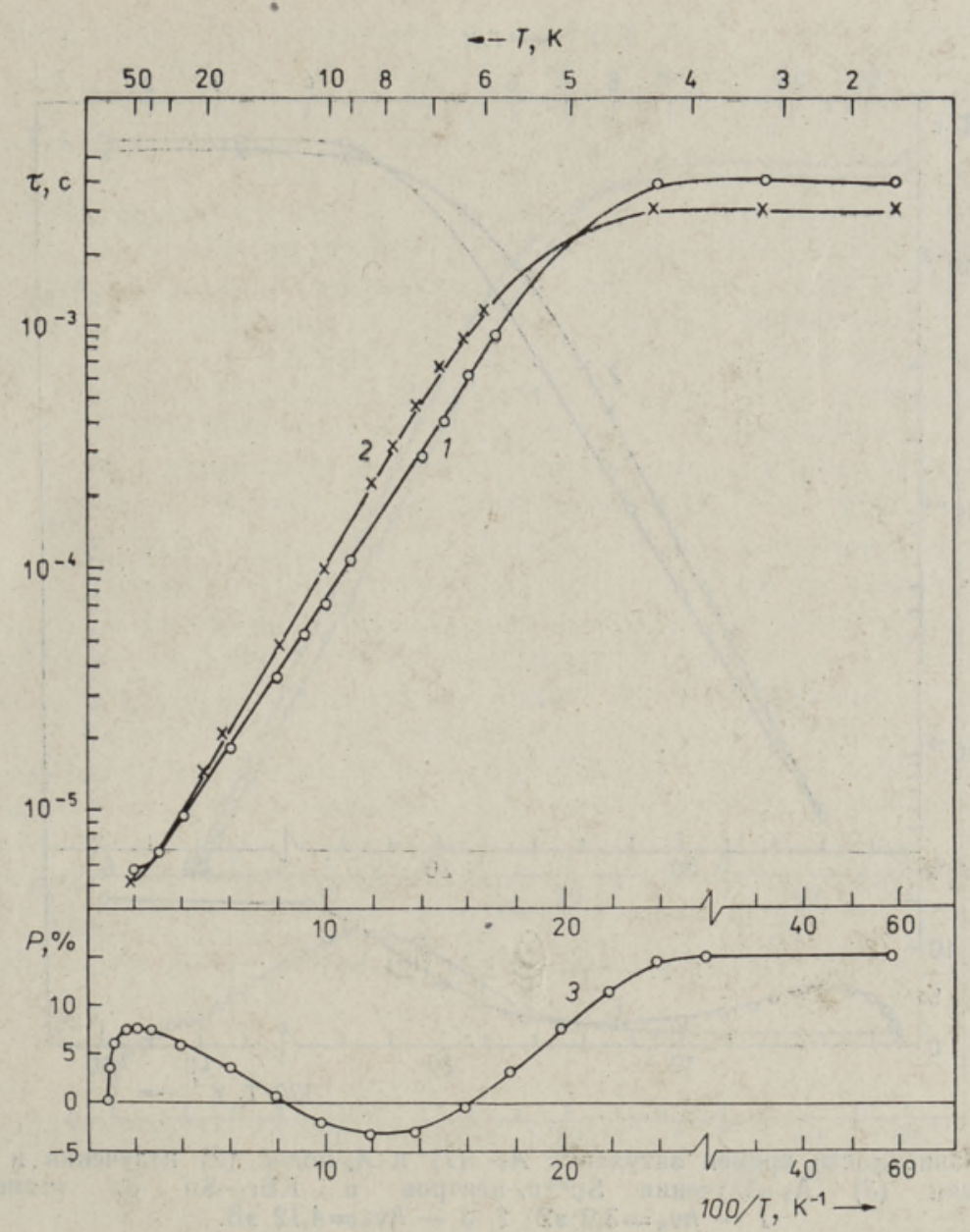

Рис. 3. Зависимости времен затухания $A_{1}$ - (1) и $A_{2}$-полос (2) излучения и степени поляризации (3) $A_{2}$-излучения $\mathrm{Sn}^{2+} v_{c}-$-центров в $\mathrm{KCl}-\mathrm{Sn}$ от температуры. $1-h v_{\mathrm{B}}=4,1{ }_{\ni} \mathrm{B} ; 2,3-h v_{\mathrm{B}}=4,5$ э $\mathrm{B}$.

из этих полос в отдельности наблюдается по одному компоненту: При $T<4,2 \mathrm{~K}$, когда излучают только метастабильные минимумы, время затухания $A_{1}$-излучения $\tau\left(A_{1}\right)$ в $1,2-1,5$ раза больше времени затухания $A_{2}$-излучения $\tau\left(A_{2}\right)$. В то же время при $T>4,2 \mathrm{~K}$, когда термически заселяются излучательные минимумы, $\tau\left(A_{1}\right)$ становится меньше $\tau\left(A_{2}\right)$. Кривая $\ln \tau\left(T^{-1}\right)$ для $A_{2}$-полосы идет несколько круче, чем длія $A_{1}$-по-

Таблица 1

Параметры Z- и X, Y-минимумов ЭПАП триплетного возбужденного состояния $\mathrm{Sn}^{2+} v_{c}$-центров в ЩГК

\begin{tabular}{|c|c|c|c|c|c|c|c|}
\hline Кристалл & $\begin{array}{l}\text { Излу- } \\
\text { чение }\end{array}$ & $\begin{array}{l}\text { Мини- } \\
\text { мум }\end{array}$ & $10^{-3}{ }^{-3} \mathrm{~B}$ & $\begin{array}{c}\gamma_{2}, \\
10^{6} \mathrm{c}^{-1}\end{array}$ & $\begin{array}{c}p_{0}, \\
10^{6} \mathrm{c}^{-1}\end{array}$ & $\begin{array}{c}y_{1}, \\
10^{3} \mathrm{c}^{-1}\end{array}$ & $g$ \\
\hline $\mathrm{KCl}-\mathrm{Sn}$ & $\begin{array}{l}A_{2} \\
A_{1}\end{array}$ & $X, Y$ & $\begin{array}{l}3,4 \\
2,9\end{array}$ & $\begin{array}{l}0,55 \\
0,4\end{array}$ & $\begin{array}{l}0,5 \\
0,4\end{array}$ & $\begin{array}{l}0,33 \\
0,26\end{array}$ & $1,50 \pm 0,05$ \\
\hline $\mathrm{KBr}-\mathrm{Sn}$ & $\begin{array}{l}A_{2} \\
A_{1}\end{array}$ & ${ }_{Z}^{X} Y$ & $\begin{array}{l}3,85 \\
3,25\end{array}$ & $\begin{array}{l}1,3 \\
0,9\end{array}$ & $\begin{array}{l}1,25 \\
0,85\end{array}$ & $\begin{array}{l}0,32 \\
0,28\end{array}$ & $1,52 \pm 0,10$ \\
\hline $\mathrm{KI}-\mathrm{Sn}$ & $\begin{array}{l}A_{2} \\
A_{1}\end{array}$ & ${ }_{Z}^{X, Y}$ & $\begin{array}{l}4,0 \\
3,6\end{array}$ & $\begin{array}{l}2,0 \\
1,8\end{array}$ & $\begin{array}{l}1,95 \\
1,8\end{array}$ & $\begin{array}{l}0,31 \\
0,28\end{array}$ & $1,52 \pm 0,05$ \\
\hline
\end{tabular}




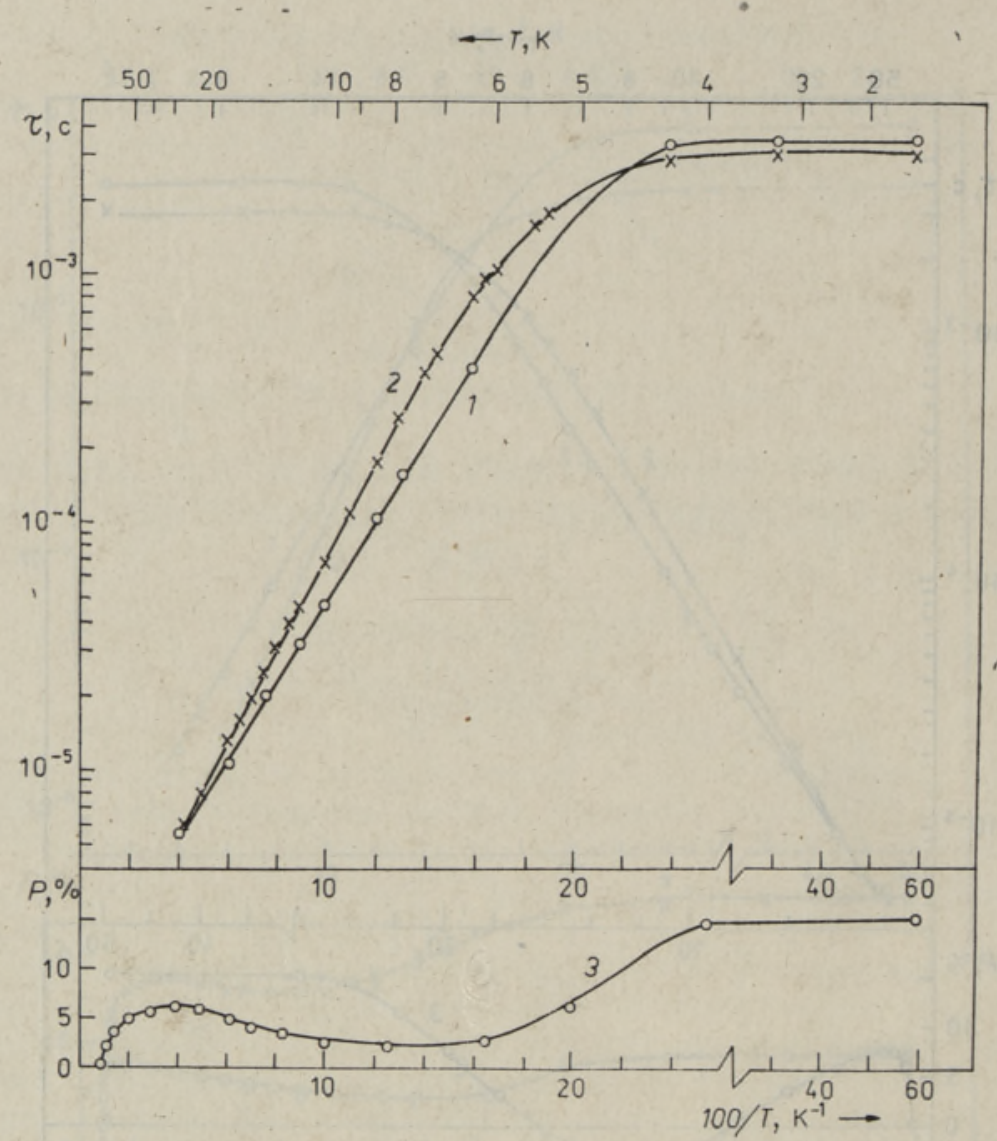

Рис. 4. Зависимости времен затухания $A_{1}$ - (1) и $A_{2}$-полос (2) излучения и степени поляризации (3) $\mathrm{A}_{2}$-излучения $\mathrm{Sn}^{2+} v_{c}$-центров в $\mathrm{KBr}-\mathrm{Sn}$ от температуры. $1-h v_{\mathrm{B}}=3,9$ эВ; $2,3-h v_{\mathrm{B}}=4,12$ эВ.

лосы. В ряду $\mathrm{KCl} \rightarrow \mathrm{KBr} \rightarrow \mathrm{KI}$ кривые $\ln \tau\left(T^{-1}\right) \quad$ как для $A_{2^{-}}$, так и для $A_{1^{-}}$ полос излучения смещаются в сторону более низких температур и наклон их увеличивается.

По формулам $\left[{ }^{16}\right]$ с использованием приведенных на рис. $3-5$ зависимостей $\ln \tau\left(T^{-1}\right)$ для $A_{2}$ и $A_{1}$-полос излучения мы рассчитали параметры $Z$ - и $X$-, $Y$-минимумов ЭПАП триплетного возбужденного состояния $\mathrm{Sn}^{2+} v_{c}^{-}$-центров (см. табл. 1). Видно, что в $Z$-минимумах величина спинового расщепления $\delta$, а также вероятности радиационного распада метастабильного $\left(\gamma_{1}\right)$ и излучательного $\left(\gamma_{2}\right)$ минимумов и безызлучательных переходов между ними $\left(p_{0}\right)$ заметно меньше, чем в $X$-, $Y$-минимумах.

В связи с вышесказанным очевидно, что в затухании суммарного $\left(A_{1}+A_{2}\right)$ излучения $\mathrm{Sn}^{2+v_{c}^{-}-ц е н т р о в ~ п р и ~ к а ж д о и ̆ ~ т е м п е р а т у р е ~ н а б л ю-~}$ даются два компонента с временами затухания $\tau\left(A_{1}\right)$ и $\tau\left(A_{2}\right)$.

\section{3. Поляризация $A_{T}$-излучения}

Условия возбуждения и наблюдения поляризованной люминесценции схематически изображены на рис. $1, a$. Степень поляризации определялась как

$$
P=\frac{I_{z}-I_{x}}{I_{z}+I_{x}}
$$




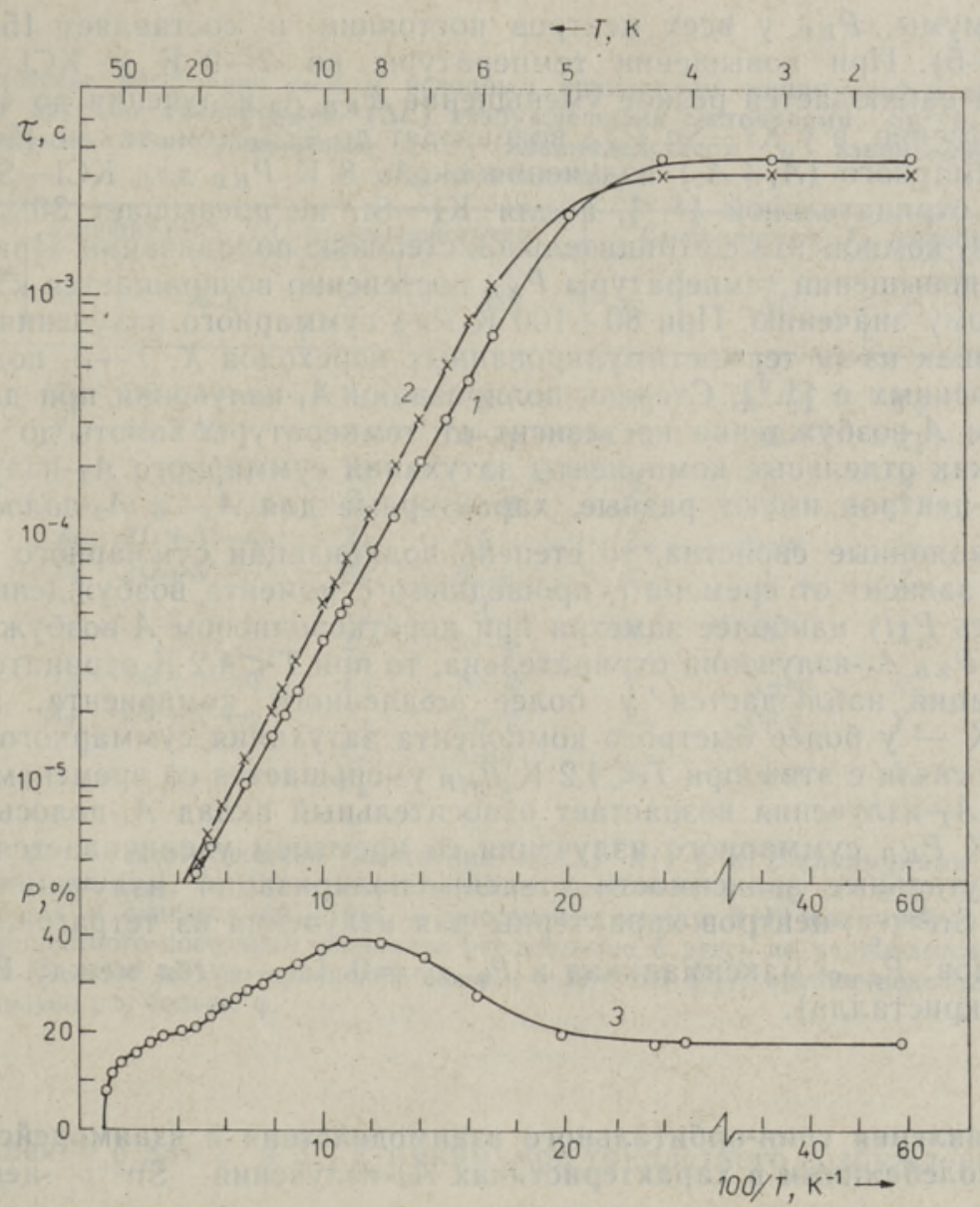

Рис. 5. Зависимости чремен затухания $A_{1}-(1)$ и $A_{2}$-полос $(2)$ излучения и степени поляризации (3) $A_{2}$-излучения $\mathrm{Sn}^{2+} v_{\mathrm{e}}{ }^{-}$-центров в $\mathrm{KI}-\mathrm{Sn}$ от температуры. $1-h v_{\mathrm{g}}=$ $=3,4$ эВ $2,3-h v_{\mathrm{B}}=3,62$ эВ.

Напомним, что в направлении $z$ излучают $X_{y}$ и $Y_{x}$ - и метастабильные

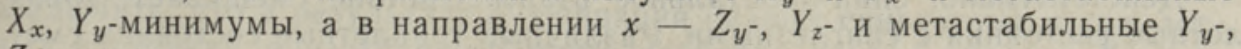
$Z_{z}$-минимумы.

Степень поляризации стационарного $A_{T}$-излучения изотропных $\mathrm{Sn}^{2+}$. центров ( $P_{\text {стац.) }}$ практически постоянна при $T<4,2 \mathrm{~K}$, когда излучают метастабильные минимумы, и составляет $50-55 \%$ в $\mathrm{KBr}-\mathrm{Sn}$ и $20-25 \%$ в $\mathrm{KCl}-\mathrm{Sn}$. При этих температурах $P$ не зависит и от времени $t$, прошедшего с момента возбуждения, вплоть до $t=6 \tau$. При повышении тем-

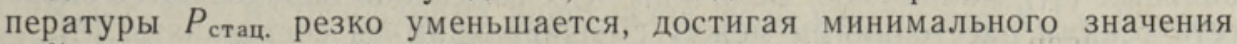
$32 \%$ у $\mathrm{KBr}-\mathrm{Sn}$ и $11 \%$ у $\mathrm{KCl}-\mathrm{Sn}$ около 12 и $7 \mathrm{~K}$ соответственно, а затем снова постепенно увеличивается (рис. 2). Около $100 \mathrm{~K}$ деполяризация излучения $\mathrm{Sn}^{2+}$-центров обусловлена термическими переходами $X$, $Y \rightleftarrows Z$.

Интересные особенности наблюдаются в поляризационных характеристиках стационарного $A_{2}$-излучения анизотропных $\mathrm{Sn}^{2+} v_{c}^{-}$-центров при возбуждении в коротковолновой (KB) области $A$-полосы поглощения, когда первоначально заселяется состояние $\frac{i}{\sqrt{2}}\left(\left|X_{y}\right\rangle-\left|Y_{x}\right\rangle\right)\left[{ }^{6}\right]$. При температурах $4,2-1,7 \mathrm{~K}$, когда излучают только метастабильные $X_{x^{-}}$, 
$Y_{y}$-минимумы, $P_{K B}$ у всех центров постоянна и составляет $15-20 \%$ (рис. 3-5). При повышении температуры до $7-9 \mathrm{~K}$ у $\mathrm{KCl}-\mathrm{Sn}$ и $\mathrm{KBr}-\mathrm{Sn}$ наблюдается резкое уменьшение $P_{K B} A_{2}$-излучения до 4 и $2 \%$ соответственно, а у KI-Sn $P_{K B}$ возрастает до $42 \%$ (см. также $\left[{ }^{4,9}, 12\right]$ ). Для суммарного $\left(A_{1}+A_{2}\right)$ излучения около $8 \mathrm{~K} P_{K в}$ для $\mathrm{KCl}-\mathrm{Sn}$ становится отрицательңой $\left[{ }^{4,9}\right]$, а для $\mathrm{KI}-\mathrm{Sn}$ не превышает $30 \%$ из-за вклада $A_{1}$-компонента с отрицательной степенью поляризации. При дальнейшем повышении температуры $P_{K в}$ постепенно возвращается к первоначальному значению. При $80-100 \mathrm{~K} P_{K B}$ суммарного излучения резко меняет знак из-за термостимулированных переходов $X, Y \rightarrow Z$, подробно рассмотренных в $\left[{ }^{4,9}\right]$. Степень поляризации $A_{1}$-излучения при длинноволновом $A$-возбуждении не зависит от температуры вплоть до $100 \mathrm{~K}$.

Так как отдельные компоненты затухания суммарного $A_{T}$-излучения $\mathrm{Sn}^{2+} v_{c}^{-}$-центров имеют разные, характерные для $A_{1}-$ и $A_{2}$-полос $\left[{ }^{2,3}\right]$ поляризационные свойства, то степень поляризации суммарного $A_{T}$-излучения зависит от времени $t$, прошедшего с момента возбуждения. 3ависимость $P(t)$ наиболее заметна при коротковолновом $A$-возбуждении. Так как $P_{K B} A_{1}$-излучения отрицательна, то при $T<4,2$ К отрицательная поляризация наблюдается у более медленного компонента, а при $T>4,2 \mathrm{~K}-$ у более быстрого компонента затухания суммарного излучения. В связи с этим при $T<4,2 \mathrm{~K} P_{K в}$ уменьшается со временем $t$, а в спектре $A_{T}$-излучения возрастает относительный вклад $A_{1}$-полосы. При $T>4,2 \mathrm{~K} P_{K B}$ суммарного излучения со временем увеличивается.

Азимутальные зависимости степени поляризации излучения всех $\mathrm{Sn}^{2+}$. и $\mathrm{Sn}^{2+} v_{c}^{-}$-центров характерны для излучения из тетрагональных минимумов: $P_{\alpha=0^{\circ}}$ максимальная и $P_{\alpha=45^{\circ}}=0\left(\alpha-\right.$ угол между $\mathbf{E}_{\text {возб. и }}$ осью $C_{4}$ кристалла).

\section{4. Проявления спин-орбитального взаимодействия и взаимодействия c $T_{2 \mathrm{~g}}$-колебаниями в характеристиках $A_{T}$-излучения $\mathrm{Sn}^{2+} v_{c}^{-}$-центров}

Особенности, наблюдающиеся в низкотемпературной кинетике затухания $A_{T}$-люминесценции анизотропных центров олова в ЩГК, можно объяснить влиянием катионной вакансии, спин-орбитального взаимодействия и взаимодействия с $T_{2 g}$-колебаниями на структуру и свойства $T$-минимумов ЭПАП триплетного возбужденного состояния ионов олова.

Действительно, спин-орбитальное взаимодействие смешивает триплетные состояния с синглетными. Различные триплетные состояния смешиваются между собой как спин-орбнтальным взаимодействием, так и

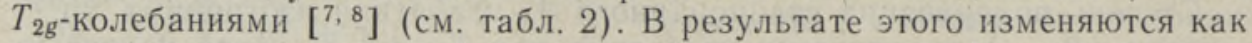
энергии разных состояний - каждый ян-теллеровский минимум расщепляется на излучательный дублет и метастабильный синглет, - так и вероятности радиационного распада излучательных $\left(\gamma_{2}\right)$ и метастабильных $\left(\gamma_{1}\right)$ минимумов. Величины этих изменений определяются энергетическими расстояниями между смешивающимися состояниями $\left[{ }^{8}\right]$.

$\mathrm{Y}$ из о т р оп ны х $\mathrm{Sn}^{2+}$-центров $\left(\varphi=0, \varphi_{0}=0\right)$ энергетические $\mathrm{pac}$ стояния практически одинаковы как между различными триплетными состояниями $(\Delta E)$, так и между различными триплетными и синглетными состояниями $\left(\Delta E_{0}\right)$, поэтому одинаковы и величины $\gamma_{2}, \gamma_{1}$ и $\delta$ для минимумов разных ориентаций.

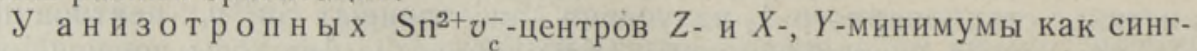
летного, так и триплетного возбужденных состояний энергетически неэквивалентны. Қак видно из табл. 2 и рис. 1, б, по этой причине для них различаются и энергетические расстояния $\Delta E_{0}$ и $\Delta E$, вследствие чего оказываются различными вероятности $\gamma_{2}$ и $\gamma_{1}$, а также спиновые 
Энергетические расстояния между исходным триплетным минимумом и синглетным $\left(\Delta E_{0}\right)$ и другими триплетными $(\Delta E)$ возбужденными состояниями $\mathrm{Sn}^{2+v_{c}-}$-центров, смешиваемыми спин-орбитальным (CO) взаимодействием и взаимодействием с $T_{2 g}$-колебаниями

\begin{tabular}{|c|c|c|c|c|c|c|c|c|}
\hline \multirow{2}{*}{$\begin{array}{l}\text { Исход- } \\
\text { ный мн- } \\
\text { нимум }\end{array}$} & \multicolumn{4}{|c|}{ Смешивается $\mathrm{CO}$-взаимодействием } & \multicolumn{4}{|c|}{ Смешивается $T_{2 g}$-колебаниями } \\
\hline & $c:$ & $\Delta E_{0}$ & c: & $\Delta E$ & c: & $\Delta E$ & c: & $\Delta E$ \\
\hline$X_{y}$ & $Z_{0}$ & $2 G+\Delta^{\prime}-\varphi_{0}$ & $Y_{x}$ & $\Delta$ & $Z_{u}$ & $\Delta-\varphi$ & $Y_{y}$ & $\Delta$ \\
\hline$X_{z}$ & $Y_{0}$ & $2 G+\Delta^{\prime}$ & $Z_{x}$ & $\Delta-\varphi$ & $Y_{z}$ & $\Delta$ & $Z_{z}$ & $\Delta-\varphi$ \\
\hline$X_{x}$ & - & - & $\begin{array}{l}Y_{y} \\
Z_{z}\end{array}$ & $\Delta \stackrel{\Delta}{-\varphi}$ & $Y_{x}$ & $\Delta$ & $Z_{x}$ & $\Delta-\varphi$ \\
\hline$Y_{x}$ & $Z_{0}$ & $2 G+\Delta^{\prime}-\varphi_{0}$ & $X_{y}$ & $\Delta$ & $Z_{x}$ & $\Delta-\varphi$ & $X_{x}$ & $\Delta$ \\
\hline$Y_{z}$ & $X_{0}$ & $2 G+\Delta^{\prime}$ & $Z_{y}$ & $\Delta-\varphi$ & $X_{z}$ & $\Delta$ & $Z_{z}$ & $\Delta-\varphi$ \\
\hline$Y_{y}$ & - & - & $\begin{array}{l}X_{x} \\
Z_{z}\end{array}$ & $\Delta \stackrel{\Delta}{\Delta}$ & $X_{y}$ & $\Delta$ & $Z_{y}$ & $\Delta-\varphi$ \\
\hline$Z_{x}$ & $Y_{0}$ & $2 G+\Delta^{\prime}+\varphi$ & $X_{z}$ & $\Delta+\varphi$ & $Y_{x}$ & $\Delta+\varphi$ & $X_{x}$ & $\Delta+\varphi$ \\
\hline$Z_{y}$ & $X_{0}$ & $2 G+\Delta^{\prime}+\varphi$ & $Y_{z}$ & $\Delta+\varphi$ & $X_{y}$ & $\Delta+\varphi$ & $Y_{y}$ & $\Delta+\varphi$ \\
\hline$Z_{z}$ & - & - & $\begin{array}{l}X_{x} \\
Y_{y}\end{array}$ & $\begin{array}{l}\Delta+\varphi \\
\Delta+\varphi\end{array}$ & $X_{z}$ & $\Delta+\varphi$ & $Y_{z}$ & $\Delta+\varphi$ \\
\hline
\end{tabular}

Здесь $\varphi, \varphi_{0}-$ энергетическне расстояния между $X(Y-)$ и $Z$-минимумами триплета $(\varphi)$ и синглета $\left(\varphi_{0}\right), \Delta, \Delta^{\prime}$ - энергетические расстояния между $X$ - и $Y$-состояниями триплета $(\Delta)$ и синглета $\left(\Delta^{\prime}\right), 2 G-$ расстояние между $X(Y)$-минимумами синглетного и триплетного состояний. Спиновое расщепление $\delta$ здесь не учитывается, так как оно мало $\left(\delta \sim 10^{-3}\right.$ эВ) по сравнению с $\varphi\left(\sim 10^{-2}\right.$ эВ $\left.\left[{ }^{6}\right]\right)$; оценки показывают, что $\varphi_{0}$ в несколько раз больше $\varphi$.

расщепления $\delta$ для $Z$ - и $X$-, $Y$-минимумов триплетного возбужденного состояния.

Особенности, наблюдающиеся в температурных зависимостях степени поляризации $A_{2}$-излучения $\mathrm{Sn}^{2+} v_{c}^{-}$-центров при коротковолновом $A$-возбуждении, можно объяснить двумя причинами: 1) расщеплением излучательного дублета в $X$ - и $Y$-минимумах и 2) туннельными переходами между $X$-, $Y$-и $Z$-минимумами.

4.1. Расщепление излучательного дублета. Анализ табл. 2 позволяет заключить, что в результате смешивания разных синглетных и триплетных состояний анизотропного ртутеподобного центра энергия $X_{y}\left(Y_{x}\right)$ минимумов может отличаться от энергии $X_{z}\left(Y_{z}\right)$-минимумов. Минимумы $Z_{x}$ и $Z_{y}$ при этом остаются энергетически эквивалентными. В зависимости от соотношения величин $\Delta, 2 G+\Delta^{\prime}, \varphi$ и фо порядок $X_{y}\left(Y_{x}\right)$ - и $X_{z}\left(Y_{z}\right)$ минимумов в разных кристаллах может быть разным. Әто позволяет объяснить зависимости степени поляризации $A_{T}$-излучения от температуры для всех исследованных кристаллов, если учесть, что $X_{y}$ и и $Y_{x}$-минимумы излучают свет, поляризованный вдоль оси $z$, а $X_{z}$ - и $Y_{z}$-вдоль $y$ и $x$ соответственно, и предположить, что в $\mathrm{KCl}-\mathrm{Sn}$ и $\mathrm{KBr}-\mathrm{Sn} X_{z}$ и $Y_{z}$-минимумы имеют меньшую, а в $\mathrm{KI}-\mathrm{Sn}$ - большую энергию, чем $X_{y^{-}}$и $Y_{x \text {-минимумы. }}$

В этом случае при коротковолновом $A$-возбуждении и $T<4,2 \mathrm{~K}$ у $\mathrm{Sn}^{2+} v_{c}^{-}$-центров первоначально заселяются метастабильные $X_{x}-, Y_{y}$-минимумы, предельная степень поляризации излучения из которых $P_{\text {пред. }}=1 / 3$. При повышении температуры заселяются нижайшие излучательные $X_{z^{-}}$и $Y_{z}$-минимумы в $\mathrm{KCl}-\mathrm{Sn}$ и $\mathrm{KBr}-\mathrm{Sn}$ и $X_{y^{-}}$и $Y_{x}$-минимумы в $\mathrm{KI}-\mathrm{Sn}$. В результате степень поляризации $A_{2}$-излучения у $\mathrm{KCl}-\mathrm{Sn}$ и $\mathrm{KBr}-\mathrm{Sn}$ резко уменьшается, а у $\mathrm{KI}-\mathrm{Sn}$ - увеличивается до экстре- 
мальных значений в области $7-9 \mathrm{~K}$ (рис. $3-5)$. При дальнейшем росте температуры по мере выравнивания заселенностей $X_{z}\left(Y_{z}\right)-$ и $X_{y}\left(Y_{x}\right)$-минимумов $P_{K в}$ стремится к прежнему значению $\left(P_{\text {пред. }}=1 / 3\right)$. Следует отметить, что данное объяснение адекватно только в том случае, если энергетическое расстояние между $X_{z}\left(Y_{z}\right)$ - и $X_{y}\left(Y_{x}\right)$-минимумами достаточно для достижения необходимой разницы их заселенностей. Существует, однако, альтернативное объяснение зависимости $P(T)$ для $A_{T}$-излучения $\mathrm{Sn}^{2+} v_{c}^{-}$-центров даже в том случае, когда расщепление излучательного дублета очень мало.

4.2. Туннельные переходы из $X$-, $Y$ - в $Z$-минимумы. В общем случае туннельные переходы между двумя данными состояниями могут разрешаться взаимодействием, смешивающим эти состояния. Поэтому переходы между состояниями типа $X_{z}$ и $Z_{x}$ разрешаются спин-орбитальным взаимодействием, а переходы между состояниями с одинаковой проекцией спиновой функции (напр., $X_{y}$ и $Z_{y}$ ) возможны благодаря взаимодействию с неполносимметричными колебаниями $\left(T_{2 g}\right.$-колебаниями в случае тетрагональных минимумов). Соответствующие вероятности туннельных переходов обозначим как $W_{C o}$ и $W_{T_{2 g}}$. В общем случае они не равны. Это означает, что вероятности туннельных переходов из двух излучательных состояний одного ян-теллеровского минимума (напр., из $X_{y}$ и $X_{z}$ ) могут быть различны.

В разных системах соотношения $\mathbb{W}_{C o}$ и $W_{T_{20}}$ могут существенно различаться в зависимости от соотношения величин спин-орбитального и янтелеровского взаимодействий. Қак видно из табл. 3, в ряду $\mathrm{KCl} \rightarrow \mathrm{KBr} \rightarrow \mathrm{KI}$ спин-орбитальное взаимодействие усиливается, а электрон-фононное относительно ослабляется. Можно предположить, что в $\mathrm{KCl}-\mathrm{Sn} W_{T_{2 g}}>W_{C O}$, а в $\mathrm{KI}-\mathrm{Sn} W_{C O}>W_{T_{2 g}}$.

В результате туннельных переходов из верхних $X$-, $Y$-минимумов в ншжние Z-минимумы *** к моменту излучения относительные заселенности $(N)$ двух спиновых компонентов излучательного дублета (напр., $X_{y}$ и $X_{z}$ ) могут оказаться различными даже в том случае, если расщепление их очень мало́. Действительно, если $W_{T_{2 g}}>W_{C o}$, то в излучательном состоянии доминируют туннельные переходы $Y_{x \rightarrow} \rightarrow Z_{x}, X_{y} \rightarrow Z_{y}$, в результате чего $N_{X_{y}, Y_{x}}$ оказывается меньше, чем $N_{X_{z}, Y_{z}}$, и $P_{K B} A_{2}$-излучения уменьшается (случай $\mathrm{KCl}-\mathrm{Sn}, \mathrm{KBr}-\mathrm{Sn}$ ). Если же $W_{C o}>W_{T_{2 g}}$, то доминируют туннельные переходы $Y_{z} \rightarrow Z_{y}, X_{z} \rightarrow Z_{x}$, из-за чего $N_{X_{y}, Y_{x}}$ оказывается больше, чем $N_{x_{z}, Y_{2}}$. и степень поляризации $A_{2}$-излучения при $K B$ возрастает (случай $\mathrm{KI}-\mathrm{Sn}$ ). При повышении температуры $\tau\left(A_{2}\right)$ резко укорачивается. В результате вероятность радиационного распада ян-теллеровских минимумов становится больше $W_{C o}\left(W_{T_{2 g}}\right)$, роль туннельных пере́ходов уменьшается и степень поляризации $A_{2}$-излучения стремится к прежнему значению $\left(P_{\text {пред. }}=1 / 3\right)$.

В случае изотропных ртутеподобных центров туннельные переходы обоих типов должны приводить к деполяризации излучения. Уменьшение степени поляризации $A_{T}$-излучения $\mathrm{Sn}^{2+}$-центров в $\mathrm{KBr}-\mathrm{Sn}$ и $\mathrm{KCl}-\mathrm{Sn}$ при повышении температуры от 4,2 до $7-12 \mathrm{~K}$ и последующее постепенное увеличение ее с температурой (рис. 2 , см. также $\left.\left[{ }^{9}\right]\right)$, напоминаю-

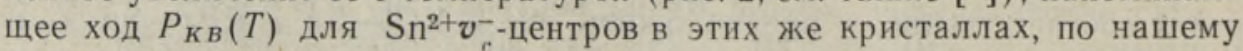
мнению, могут быть проявлением туннельных переходов между излучательными минимумами разных ориентаций.

Туннельные переходы из $X-, Y$ - в $Z$-минимумы могут оказывать влияние и на низкотемпературную кинетику затухания $A_{T}$-излучения

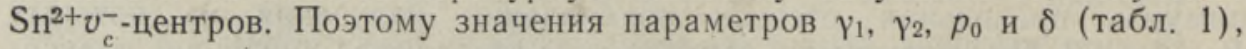

\footnotetext{
*** Вероятность обратных переходов $Z \rightarrow X, Y$ прн температурах $T<100 \mathrm{~K}$ пренебре-
} жимо мала. 
Параметры, характеризующие спин-орбитальное $(R, \delta)$ и электрон-фононное $\left(S, \delta_{m}{ }^{1}\right)$

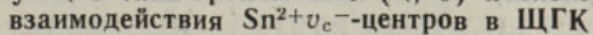

\begin{tabular}{l|ll|lll|l}
\hline Кристалл & & $R$ & & $\delta, 10^{-2}{ }^{\text {эB }}$ & $S$, эВ & $\delta_{m}{ }^{\mathrm{I}},{ }^{\mathrm{B}}$ \\
\hline $\mathrm{KCl}-\mathrm{Sn}$ & 18 & ${ }^{\left[{ }^{19}\right]}$ & 0,308 & $\left.{ }^{16}\right]$ & 1,65 & 0,29 \\
$\mathrm{KBr}-\mathrm{Sn}$ & 15,4 & {$\left[{ }^{17}\right]$} & 0,335 & {$\left[{ }^{16}\right]$} & 1,31 & 0,22 \\
$\mathrm{KI}-\mathrm{Sn}$ & 11 & {$\left[{ }^{18}\right]$} & 0,36 & {$\left[{ }^{16}\right]$} & 1,07 & 0,18
\end{tabular}

$R$ - соотношение сил осцилляторов $C$ - и $A$-полос поглощения,

$\delta$ - спиновое расщепление $T$-минимумов,

$S$ - стоксовые потери,

$\delta_{m}$ I - полуширина полосы $A_{2}$-излучения при $4,2 \mathrm{~K}$.

определенные по формулам $\left[{ }^{16}\right]$, не учитывающим туннельных переходов, могут несколько отличаться от истинных. Возможно из-за этого у всех $\mathrm{Sn}^{2+} v_{c}^{-}$-центров величина g-фактора (табл. 1), вычисленная с использованием этих параметров (см. также $\left[{ }^{20}\right]$ ) оказывается гораздо меньше теоретической $\left(g=2\left[{ }^{7,8}\right]\right)$. Не исключена и возмажность нецентрального положения иона $\mathrm{Sn}^{2+}$ в галогенидах калия, что тоже может существенно сказываться на кинетике затухания излучения.

Таким образом, особенности, наблюдаемые в низкотемпературной кинетике затухания $A_{T}$-излучения $\mathrm{Sn}^{2+} v_{c}^{-}$-центров в ЩГК, объясняются различием параметров $\left(\delta, \gamma_{2}, \gamma_{1}\right) Z$ - и $X$-, $Y$-минимумов ЭПАП триплетного возбужденного состояния. Последнее вызвано энергетической неэквивалентностью $Z$ - и $X$-, $Y$-минимумов как триплетного, так и синглетного состояний, в результате чего к разным триплетным минимумам в разной степени подмешиваются спин-орбитальным взаимодействием и $T_{2 g}$-колебаниями синглетные и другие триплетные состояния. Это приводит и к возникновению более тонкой структуры ЭПАП триплетного возбужденного состояния, в частности, к расщеплению излучательного дублета в $X$ - и $Y$-минимумах.

Особенности, наблюдаемые в температурных зависимостях степени поляризации $A_{2}$-излучения $\mathrm{Sn}^{2+} v_{\mathrm{c}}^{-}$-центров при низких температурах, могут быть обусловлены двумя, по нашему мнению, равновероятными причинами: 1) вышеупомянутым расщеплением излучательного дублета и 2) туннельными переходамй из верхних $X$-, $Y$ - и нижние $Z$-минимумы. $\mathrm{B} \mathrm{KCl}-\mathrm{Sn}$ и $\mathrm{KBr}-\mathrm{Sn}$ в этом случае должны доминировать туннельные переходы, обусловленные $T_{2 g}$-колебаниями, а в $\mathrm{KI}-\mathrm{Sn}-$ спин-орбитальным взаимодействием.

Авторы выражают глубокую признательность В. В. Хижнякову за полезные дискуссии и И. Долиндо за помощь в проведении некоторых экспериментов.

\section{Л ИТЕРА Т У Р}

1. Зазубович С. Г. Оптика и спектр., 37, вып. 4, 711-716 (1974).

2. Зазубович С. Г. Тр. ИФ АН ЭССР, 23, 38-59 (1963).

3. Lushchik, N., Zazubovich, S. In: Physics of Impurity Centres in Crystals (Ed. by G. Zavt). Tallinn, 1972, 483-504. 
4. Hizhnyakov, V., Zazubovich, S., Soovik, T. Phys. status solidi (b), 66, № 2, 727732 (1974).

5. Тринклер Л. Е., Тринклер М. Ф. Изв. АН Латв. ССР, сер. физ. и техн. наук, 3, 33-39 (1980).

6. Hizhnyakov, V., Zazubovich, S. Phys. status solidi (b), 86, № 2, 733-739 (1978).

7. Хижняков В. В. Препринт FI-36. Тарту, 1975.

8. Hizhnyakov, V., Kristoffel, N. In: The Dynamical Jahn-Teller Effect in Localized Systems (Ed. by Yu. E. Perlin, M. Wagner). Elsevier Sci. Publishers B. V., North-Holland, 1984, 383-438.

9. Зазубович С. Г., Соовик T. А., Хижняков В. В. Изв. АН СССР. Сер. физ., 40, вып. 9, 1944-1948 (1976).

10. Kamishina, Y., Jacobs, P. W. M., Simkir, D. J., Martin, J. P., Oyama-Gannon, K., Le Si Dang. Phys Rev. B, 22, № 6, 3010-3020 (1980).

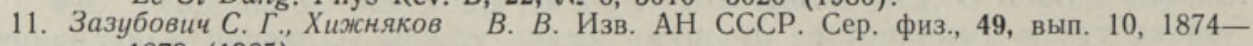
1879 (1985).

12. Le Si Dang, Simkin, D. J., Jacobs, P. W. M. J. Luminescence, 24/25, 205-208 (1981).

13. Sivasankar, V. S., Scacco, A., Schmitt, K., Jacobs, P. W. M., Simkin, D. J. Rad. Effects, 73, 77-80 (1983).

14. Le Si Dang, Jacobs, P. W. M., Schmitt, K., Sivasankar, V. S., Simkin, D. J. J. Chem. Phys., 66, 51-55 (1982).

15. Simkin, D. J., Gannon, K. O., Martin, J. P., Kamishina, Y., Jacobs, P. W. M. J. Luminescence, 18/19, 623-628 (1979).

16. Зазубович С. Г., Нагирный В. П., Соовик Т. А. Тр. ИФ АН ЭССР, 58, 181-190 (1986).

17. Gannon, K. O., Jacobs, P. W. M. J. Phys. Chem. Solids, 36, 1375-1382 (1975).

18. Sivasankar, V. S., Kamishina, Y., Jacobs, P. W. M. J. Chem. Phys., 76, № 10 , 4681-4688 (1982).

19. Honma, A. Sci. Light, 23, № 2, 43-48 (1974).

20. Брагина Л. А., Зазубович С. Г.. Лийдья Г. Г., Нагирный В. П., Соовик Т. А. Тр. ИФ АН ЭССР, 58, 169-180 (1986).

$\begin{array}{cc}\text { Институт физики } & \text { Поступила в редакцию } \\ \text { Академии наук Әстонской ССР } & 16 / \mathrm{VI} 1986\end{array}$

Svetlana ZAZUBOVITS, N. NAGIRNOI, T. SOOVIK

\section{KAHEVALENTSETE TINATSENTRITE TRIPLETSE LUMINESTSENTSI KUSTUMIS- KINEETIKA JA POLARISATSIOON LEELISHALOGENIIDKRISTALLIDES}

On uuritud rea leelishalogeniidkristallide isotroopsete $\mathrm{Sn}^{++}$-tsentrite $A_{T}$-kiirguse

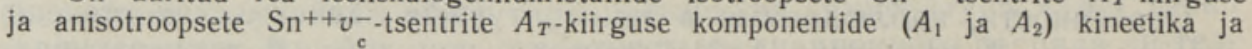
polarisatsiooni karakteristikuid temperatuurivahemikus $1,7-40 \quad \mathrm{~K}$. Sn $\mathrm{Sn}^{++v_{c}^{-}-\text {tsentrite }}$ $A_{1}$ ja $A_{2}$ kiirgusribade vahel ilmnesid märgatavad erinevused kustumisaegades ning kustumisaegade ja polarisatsiooniastme temperatuurilises käigus. Need efektid on sele-

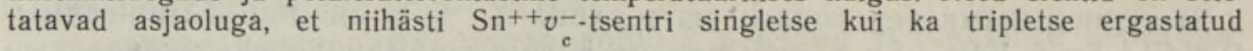
seisundi $Z$ - ja $X, Y$-miinimumide energeetilise mitteekvivalentsuse tõttu erinevad tripletsed miinimumid segunevad spinnorbitaalse vastastikmõju ja $T_{2 g}$-võnkumiste toimel singletsete ja teiste tripletsete seisunditega erineval määral. Tulemusena osutuvad erinevaks tripletsete $Z$ - ja $X, Y$-miinimumide kiirgusliku lagunemise tõenäosused ja spinnlōhenemised ning lōheneb kiirgav dublett $X, Y$-miinimumides.

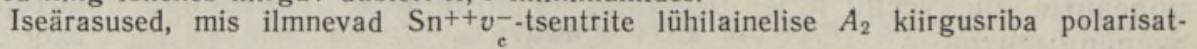
siooniastme temperatuurilises olenevuses, võivad olla seotud ka tunnelüleminekutega ülemistest $X, Y$-miinimumidest alumistesse $Z$-miinimumidesse, mis on pöhjustatud kas vastastikmõjust $T_{2 g}$-vōnkumistega $(\mathrm{KCl}-\mathrm{Sn}, \mathrm{KBr}-\mathrm{Sn})$ või spinnorbitaalsest interaktsioonist. 


\section{DECAY KINETICS AND POLARIZATION OF THE TRIPLET LUMINESCENCE OF DIVALENT TIN CENTRES IN ALKALI HALIDES}

Low temperature decay kinetics and polarization are investigated for the $A_{T}$ emission of isotropic $\mathrm{Sn}^{2+}$ centres and for separate components $\left(A_{1}, A_{2}\right)$ of the $A_{T}$ emission of anisotropic $\mathrm{Sn}^{2+v_{c}^{-}}$centres in alkali halides. The decay times $\tau$ and their temperature dependences $\tau(T)$ as well as the temperature dependences of the polarization degree $P(T)$ are found to be different for the $A_{1}$ and $A_{2}$ emission bands of $\mathrm{Sn}^{2+} v_{c}^{-}$.

The effects are explained as follows. As the $Z$ and $X, Y$ minima of both the singlet and triplet excited states of $\mathrm{Sn}^{2+v_{c}^{-}}$centres have different energies, the mixing of the triplet minimum with singlet or other triplet states by the spin-orbit interaction or $T_{2 g}$ vibrations is different for $Z$ and $X, Y$ minima. As a result, the probabilities of the radiative decay of emitting and metastable minima and the energy distance between them become different for the triplet $Z$ and $X, Y$ minima. The splitting of the emitting doublet in $X, Y$ minima is also evident. The tunnel transitions form the upper $(X, Y)$ to the lower $(Z)$ minima of the triplet state, caused by $T_{28}$ vibrations ( $\mathrm{KCl}: \mathrm{Sn}$, $\mathrm{KBr}: \mathrm{Sn})$ or by spin-orbit interaction (KI:Sn), may also be responsible for the unusual dependences $\mathrm{P}(\mathrm{T})$ observed for the high-energy $\left(A_{2}\right)$ emission band of $\mathrm{Sn}^{2+v} v_{c}^{-}$ centres. 